\title{
Distributed Policy Management in Semantic Web
}

\author{
Özgü Can and Murat Osman Ünalır \\ Department of Computer Engineering, Ege University, \\ 35100 Bornova, İzmir, Turkey \\ \{ozgu.can, murat.osman.unalir\}@ege.edu.tr
}

\begin{abstract}
With the growth of Internet and the fast development of semantic web technologies, the access and usage of information will become much more easier. However, the security of information gathering for both information suppliers and demanders is a critical issue. Policies determine the ideal behaviors for web concepts. The concepts of policy, policy languages and policy ontologies must be determined, effective tools for policy definition and management must be developed.
\end{abstract}

\section{Introduction}

In the web environment some concepts are very important: which resources can users or the services access (security), how will the user information going to be used (privacy) and if these users are trusted or not (trust). Policies specify who can use a service and under which conditions, how information should be provided to the service, and how the provided information will be used [1]. Two main components exist to secure the semantic web: a policy language which defines security requirements and distributed policy management approach [2]. A security framework based on distributed policy management can be developed with using a semantic policy language.

In the literature, there are various policy ontology and policy language projects. The most popular ones are $\mathrm{KAoS}^{1}, \mathrm{Rei}^{2}$ and Ponder $^{3}$ policy languages, and a framework, Rein ${ }^{4}$, which uses the Rei policy language concepts for policies.

\section{Research Approach}

In our work, we are going to examine existing policy ontologies, KAoS, Rei and Ponder, then we will determine the requirements of policy ontologies and the missing points of the existing policy ontologies. Through these requirements we will constitute a new policy ontology. Our aim is to create an effective new policy ontology which overcomes the missing points of the existing policies and answers the

\footnotetext{
${ }^{1}$ http://ontology.ihmc.us/kaos.html

${ }^{2} \mathrm{http}: / /$ rei.umbc.edu/

${ }^{3}$ http://www-dse.doc.ic.ac.uk/Research/policies/ponder.shtml

${ }^{4}$ http://groups.csail.mit.edu/dig/2005/05/rein/
} 
user's requests in a better manner. Authorizations and obligations are the policy concepts in KAoS; rights, prohibitions, obligations and dispensations are Rei policy language concepts and Ponder has authorizations, obligations, refrains and delegations. In the policy ontology we will have rights, prohibitions, obligations, dispensations and speech acts: delegate, revoke, cancel and request.

One of the existing works is a plug-in application, POLICYTAB [3], which is compatible with Protégé. In this thesis, we want to develop an OWL based plug-in for Protégé Ontology Editor to define policy specifications. Policy rules will be expressed by using Semantic Web Rule Language (SWRL). We will also develop a policy engine to interpret and reason over policies.

There are three policy approaches: rule-based, ontology-based and hybrid [4]. We are going to use hybrid approach in our work. Hybrid approach is based on both rulebased and ontology-based approaches. We will also focus on policy conflicts and policy validation.

Our ontology will be developed with OWL ontology language on Protégé ontology editor. We will use SWRL for rules, RACER reasoning engine, OWL-QL for queries and Java.

\section{Conclusions and Future Work}

Our prerequisite work is to create our policy ontology and to work on a resource sharing scenario where policies can be applied. Our scenario is based on our university's academic rules and regulations. Now we are constituting our ontology concepts. After that we are going to create our ontology by using Rei. We also want to develop our policy engine and effective mechanisms to solve policy conflicts. Finally we will work on policy validation methods.

\section{References}

1. L. Kagal, T. Finin, M. Paolucci, N. Srinivasen, K. Sycara, G. Denker: Authorization and Privacy for Semantic Web Services. IEEE Intelligent Systems, Vol. 19 (2004) 50-56

2. L. Kagal, T. Finin, A. Joshi: A Policy Based Approach to Security for the Semantic Web. $2^{\text {nd }}$ International Semantic Web Conference (ISWC 2003), Sanibal Island, Florida, USA (2003) 402-418

3. W. Nejdl, D. Olmedilla, M. Winslett, C. C. Zhang; Ontology-based Policy Specification and Management. European Semantic Web Conference (ESWC 2005), Heraklion, Greece (2005)

4. A. Toninelli, J. M. Bradshaw, L. Kagal, R. Montanari; Rule-based and Ontology-based Policies: Toward a Hybrid Approach to Control Agents in Pervasive Environments. $4^{\text {th }}$ International Semantic Web Conference, Proceedings of the Semantic Web and Policy Workshop, Galway, Ireland (2005) 